\title{
Concurrent Verbal Encouragement and Wingate Anaerobic Cycle Test Performance in Females: Athletes vs. Non-Athletes
}

\author{
D.L. Bullinger*, C.M. Hearon FACSM, S.A. Gaines and M.L. Daniel \\ Department of Health \& Kinesiology \\ Texas A\&M University-Kingsville \\ Kingsville, TX \\ (*undergraduate)
}

Previous research has shown that concurrent verbal encouragement improves performance on the Wingate Anaerobic Cycle Test (WACT), a 30-s supramaximal exercise protocol, in male non-athletes. This effect has not previously been examined in athletes or females. Consistently, athletes and females display a more self-determined motivational profile than non-athletes and males, suggesting verbal encouragement to be of lesser consequence to athletes and females. PURPOSE: To examine the effect of concurrent verbal encouragement on the performance of the WACT in female athletes vs. female non-athletes. METHODS: Nineteen college-age female subjects were recruited where ten of the subjects were active intercollegiate athletes (ATH, $\left.n_{1}=10\right)$ and the remainder were non-athletes (NON, $n_{2}=9$ ). The WACT was novel to all subjects and the subjects were blinded to the purpose of the study. Prior to participation in the experimental trials, subjects were measured for body composition and performed a familiarity WACT trial without verbal encouragement. Subjects then performed the WACT twice, once with concurrent verbal encouragement (VE) and once without (NVE), in a balanced cross-over design. All WACT trials were performed at least one week apart. Peak power (PP), mean power (MP), and total work (TW) were compared between ATH and NON across VE and NVE using an ANOVA (1 between, 1 within), $\alpha=0.05$. RESULTS: ATH and NON did not differ significantly $(p>0.05)$ with regard to age or body composition with the exception of fat-free mass which differed significantly $(\mathrm{ATH}=53.7 \pm 6.6, \mathrm{NON}=46.1 \pm 5.7 \mathrm{~kg})(p<0.05)$. As expected, a significant $(p<0.05)$ main effect for ATH/NON was observed where ATH outperformed NON when pooled across $\mathrm{VE} / \mathrm{NVE}$ trials for $\mathrm{PP}\left(\mathrm{ATH}=13.0 \pm 1.4, \mathrm{NON}=11.3 \pm 1.7 \mathrm{~W} \cdot \mathrm{kg}^{-1}\right), \mathrm{MP}(\mathrm{ATH}=7.7 \pm 1.1$, $\left.\mathrm{NON}=6.7 \pm 0.9 \mathrm{~W} \cdot \mathrm{kg}^{-1}\right)$ and TW $\left(\mathrm{ATH}=232 \pm 35, \mathrm{NON}=201 \pm 26 \mathrm{~J} \cdot \mathrm{kg}^{-1}\right)$. However, when pooled across all subjects (ATH and NON), the VE/NVE trials did not differ significantly $(p>0.05)$ for $\mathrm{PP}\left(\mathrm{VE}=12.4 \pm 1.7, \mathrm{NVE}=12.0 \pm 1.9 \mathrm{~W} \cdot \mathrm{kg}^{-1}\right), \mathrm{MP}(\mathrm{VE}=7.3 \pm 1.1, \mathrm{NVE}=7.2 \pm 1.2$ $\left.\mathrm{W} \cdot \mathrm{kg}^{-1}\right)$ and TW $\left(\mathrm{VE}=219 \pm 33, \mathrm{NVE}=215 \pm 35 \mathrm{~J} \cdot \mathrm{kg}^{-1}\right)$. Additionally, the ATH/NON interaction with VE/NVE was not significant $(p>0.05)$. CONCLUSION: Concurrent verbal encouragement does not affect performance on the WACT in females, nor does it affect WACT performance in female athletes and non-athletes differently. 